\title{
The Pacific-specific CREBRF rs373863828 allele protects against gestational diabetes mellitus in Māori and Pacific women with obesity
}

\author{
Mohanraj Krishnan ${ }^{1,2} \cdot$ Rinki Murphy ${ }^{2,3,4}$ - Karaponi A. M. Okesene-Gafa ${ }^{1,3} \cdot$ Maria Ji ${ }^{1}$ J John M. D. Thompson ${ }^{1,5}$. \\ Rennae S. Taylor ${ }^{1}$ • Tony R. Merriman ${ }^{4,6}$ • Lesley M. E. McCowan ${ }^{1,3} \cdot$ Christopher J. D. McKinlay $^{3,7}$ (D)
}

Received: 29 October 2019 / Accepted: 6 May 2020 / Published online: 12 July 2020

(C) Springer-Verlag GmbH Germany, part of Springer Nature 2020

\begin{abstract}
Aims/hypothesis The CREBRF rs 373863828 minor (A) allele is associated with increased BMI but reduced prevalence of type 2 diabetes in Māori and Pacific people. Given the shared aetiology of type 2 diabetes and gestational diabetes mellitus (GDM), we tested for an association between the $C R E B R F$ rs373863828 variant and GDM.

Methods We conducted a prospective cohort study of Māori and Pacific women nested within a nutritional intervention study for pregnant women with obesity. Women were enrolled at 12-17 weeks' gestation and underwent anthropometry and collection of buffy coats for later genetic testing. GDM was diagnosed by 75 g OGTT at 24-28 weeks' gestation using the International Association of Diabetes and Pregnancy Study Groups criteria. Genotyping was performed by real-time PCR with a custom $C R E B R F$ rs 373863828 probe-set. The association between $C R E B R F$ rs373863828 and GDM was analysed separately by ethnic group using logistic regression, with effect estimates combined in a meta-analysis.

Results Of 112 Māori and Pacific pregnant women with obesity, 31 (28\%) carried the CREBRF rs373863828 A allele (A/G or $\mathrm{A} / \mathrm{A})$ and $35(31 \%)$ developed GDM. Women who carried the $C R E B R F$ rs373863828 A allele did not differ in BMI when compared with non-carriers $(\mathrm{G} / \mathrm{G})$. There was a fivefold reduction in the likelihood of GDM per $C R E B R F \mathrm{rs} 373863828$ A allele (OR 0.19 [95\% CI 0.05, 0.69], $p=0.01$ ), independent of age, BMI and family history of diabetes (adjusted OR 0.13 [95\% CI $0.03,0.53], p=0.004)$. GDM was diagnosed in $10 \%$ and $40 \%$ of women with and without the CREBRF rs $373863828 \mathrm{~A}$ allele, respectively (no woman with the A/A genotype developed GDM).

Conclusions/interpretation The CREBRF rs373863828 (A) allele is associated with reduced likelihood of GDM in Māori and Pacific women with obesity and may improve GDM risk prediction.
\end{abstract}

Keywords $C R E B R F \cdot$ Gestational diabetes mellitus $\cdot$ Maternal obesity $\cdot$ Type 2 diabetes mellitus

\section{Abbreviations}

GDM Gestational diabetes mellitus

GWAS Genome wide association studies

GWG Gestational weight gain

Christopher J. D. McKinlay

c.mckinlay@auckland.ac.nz

Rinki Murphy

r.murphy@auckland.ac.nz

1 Department of Obstetrics and Gynaecology, University of Auckland, Auckland, New Zealand

2 Department of Medicine, University of Auckland, Private Bag 92019, Auckland 1142, New Zealand

3 Counties Manukau Health, Auckland, New Zealand
HUMBA Healthy Mums and Babies

IADPSG International Association of Diabetes and Pregnancy Study Groups

ROC Receiver operating characteristic

4 Maurice Wilkins Centre for Molecular Biodiscovery, University of Auckland, Auckland, New Zealand

5 Department of Paediatrics, University of Auckland, Auckland, New Zealand

6 Department of Biochemistry, University of Otago, Dunedin, New Zealand

7 Liggins Institute, University of Auckland, Private Bag 92019, Auckland 1142, New Zealand 


\section{Research in context}

\section{What is already known about this subject?}

- The CREBRF rs373863828 A (minor) allele is associated with increased BMI but reduced risk of type 2 diabetes in Māori and Pacific people

- Gestational diabetes mellitus (GDM) and type 2 diabetes have a shared pathophysiology

- New approaches are needed to identify women at higher or lower risk of GDM so that pre-conceptual and early pregnancy interventions can be employed to prevent glucose intolerance

What is the key question?

- Is the CREBRF rs373863828 A (minor) allele associated with risk of GDM in Māori and Pacific women with obesity?

\section{What are the new findings?}

- The CREBRF rs373863828 A (minor) allele is carried by $28 \%$ of Māori and Pacific women with obesity

- This allele is associated with a fivefold reduction in the likelihood of GDM in Māori and Pacific women with obesity

- The addition of CREBRF rs373863828 status improved the predictive value of known clinical risk factors for GDM

How might this impact on clinical practice in the foreseeable future?

- Knowledge of CREBRF rs373863828 genotype may improve GDM risk prediction in Māori and Pacific women

\section{Introduction}

Gestational diabetes mellitus (GDM) is an increasing health problem worldwide and is associated with short- and longterm health risks for women and their offspring [1]. GDM is a state of impaired glucose tolerance and/or increased fasting blood glucose concentrations first recognised in pregnancy [2]. Normal pregnancy is characterised by increasing peripheral insulin resistance, thereby promoting transfer of glucose and fatty acids from the mother to the fetus. These changes are exaggerated in women with GDM; raised postprandial glucose concentration and/or fasting hyperglycaemia ensue when pancreatic insulin secretion is insufficient to achieve adequate glucose clearance and suppression of hepatic glucose output, respectively [2]. This may reflect an underlying defect in insulin signalling or pancreatic function [3].

Although there is ongoing debate about optimal methods for screening and diagnosis of GDM, the International Association of Diabetes and Pregnancy Study Groups (IADPSG) has recommended universal testing of pregnant women using an OGTT at 24-28 weeks of gestation [4]. However, the logistical challenges of completing an OGTT, with the required overnight fasting, blood sampling over $2 \mathrm{~h}$ and frequent nausea, impacts on uptake and adequacy of testing and GDM detection, while the timing of the OGTT results in a narrow window of opportunity for intervention in the third trimester. Further, there is increasing evidence that women with GDM may have metabolic derangement from early in pregnancy prior to clinically detected glucose intolerance [5]. Thus, an evidence-based strategy that enables risk stratification for GDM in early pregnancy or even prior to conception would be beneficial.

The genetic contribution to GDM is an emerging area of research that could potentially inform future risk stratification. Numerous genome wide association studies (GWAS) have identified strong reproducible susceptibility variants for type 2 diabetes across different populations [6-8]. In Korean women, GWAS have identified variants in $C D K A L 1$ and near MTNR1B that are associated with GDM [9]. Other studies have found that several variants (including $C D K A L 1$ and near $M T N R 1 B)$ are associated with increased risk of both type 2 diabetes and GDM, suggesting that these conditions may have a shared genetic background $[10,11]$. This is further supported by the fact that women affected by GDM have up to a sevenfold increased risk of subsequently developing type 2 diabetes [12].

Recently, a missense variant (rs373863828, Arg457Gln, c. $1370 \mathrm{G}>\mathrm{A})$ in the $C R E B R F$ gene was identified as being strongly associated with higher BMI $\left(+1.4 \mathrm{~kg} / \mathrm{m}^{2}\right)$ and waist circumference $(+3 \mathrm{~cm})$, but lower risk of type 2 diabetes (OR 0.59) among adults of Polynesian [13, 14] and Micronesian [15] ancestry. The minor (A) allele is prevalent in New Zealand Māori and Pacific people (allelic frequency 10$27 \%$ ) but exceedingly rare in other ethnic groups $(0.01 \%$ in East Asians and $0.004 \%$ in Europeans) in the Genome Aggregation Database (http://gnomad.broadinstitute.org, accessed 1 October 2017) [14]. It is currently not known whether the CREBRF rs373863828 (A) variant is associated with reduced risk of GDM. Thus, our aim was to examine the association of the $C R E B R F$ missense variant with risk of 
GDM in Māori and Pacific women with obesity and to investigate whether the CREBRF variant genotype improves clinical risk prediction for GDM in early pregnancy within this subgroup of Māori and Pacific women.

\section{Methods}

Study population This study was undertaken among women recruited to a nutritional intervention study known as the Healthy Mums and Babies (HUMBA) trial (www.anzctr.org. au registration no. ACTRN12615000400561) in South Auckland, New Zealand, where more than half of the maternity population is of Māori and Pacific descent [16]. The HUMBA trial is a $2 \times 2$ factorial randomised controlled trial that investigated whether excessive gestational weight gain in pregnant women with obesity and birthweight in their infants could be reduced by the following interventions: (1) a multi-faceted dietary intervention provided by community health workers that included text messaging compared with routine dietary advice; and/or (2) probiotics compared with placebo. The dietary intervention consisted of four home-based education sessions, reinforced with behaviour change techniques, personalised pregnancy weight gain targets and motivational text messaging three times per week. Women allocated to routine dietary advice received routine pamphlets on healthy eating in pregnancy available to all women in New Zealand. Women allocated to probiotics received a capsule of Lactobacillus rhamnosus $G G$ and Bifidobacterium lactis BB12 (Chr.Hansen, Denmark) at a dose of $7 \times 10^{9}$ colony forming units daily until birth.

Women were eligible for this genetic substudy if they had a grandparent of New Zealand Māori or Pacific (Polynesian) ethnicity as determined by maternal self-report. If women had both Māori and Pacific ancestry, ethnicity was prioritised as Māori, in line with Statistics New Zealand guidelines. Women with a singleton pregnancy and BMI $\geq 30 \mathrm{~kg} / \mathrm{m}^{2}$ were recruited to the HUMBA trial between $12^{+0}$ and $17^{+6}$ weeks of gestation (gestational age is given as weeks ${ }^{\text {days }}$ ). Women with pre-existing diabetes $\left(\mathrm{HbA}_{1 \mathrm{c}} \geq 50 \mathrm{mmol} / \mathrm{mol}[\geq 6.7 \%]\right)$ in early pregnancy were excluded [17].

Ethics approval for this study was obtained from the Southern Health and Disability ethics committee, New Zealand (14/STH/205). All participants provided written informed consent for trial participation, the collection of samples and subsequent genetic analysis.

Measurement of variables Data obtained at the recruitment visit included demographic data, family history of diabetes, maternal anthropometric measures (height, weight, waist circumference and mid-arm circumference) and BP. Anthropometric measures were repeated at 28-30 weeks' and 36 weeks' gestation. Gestational weight gain (GWG) was defined as mean weekly weight gain between recruitment to 28 and 36 weeks' gestation by Institute of Medicine criteria [18]. Finger-prick non-fasting blood lipid and $\mathrm{HbA}_{1 \mathrm{c}}$ testing was conducted at each study visit (recruitment,) using the Roche Cobas b 101 point-of-care system [19]. A non-fasting blood (buffy coat) specimen was collected for genetic testing in consenting women.

Participants underwent a 75 g OGTT at 24-28 weeks' gestation before the second trial visit, including fasting, $1 \mathrm{~h}$ and $2 \mathrm{~h}$ venous blood glucose measurements. In this study, GDM was defined by IADPSG criteria (glucose concentrations: fasting $\geq 5.1 \mathrm{mmol} / 1 ; 1 \mathrm{~h} \geq 10 \mathrm{mmol} / 1$; or $2 \mathrm{~h}$ $\geq 8.5 \mathrm{mmol} / \mathrm{l}$ ) [4]. It is unlikely that the women developed GDM after the 24-28 week timepoint, as pregnancy-related increases in insulin resistance peak by 24 weeks' gestation. Neither of the trial interventions (dietary intervention vs routine dietary advice nor probiotics vs placebo) altered the incidence rate of GDM, and so groups were combined for analysis.

SNP design and genotyping DNA extraction was conducted according to the manufacturer's recommendation using the PureLink Genomic DNA Mini Kit (Invitrogen, USA). A custom designed TaqMan probe-set (Applied Biosystems, USA) was created for rs373863828 using a custom Python script (snp_design; DOI: https://doi.org/10.5281/zenodo. $56250)$ to annotate the human genome build 37 reference sequence (ftp://ftp.ensembl.org/pub/grch37, accessed 1 August 2016) with rs373863828 and any surrounding SNPs (obtained from the NCBI dbSNP build 147 common SNP list; ftp://ftp.ncbi.nlm.nih.gov/snp): forward primer: CAAGAGAGGATGCTGAGACCAT; reverse primer: ACCATGATGTAAGCCATTTTTCTGATACA; probe 1 (VIC): TGAGTGGAACCGAGATAC probe 2 (FAM): AGTGGAACCAAGATAC. Genotyping was performed using the LightCycler 480 Real-Time PCR System in 384well plates (Roche Applied Science, USA). Quality control measures included genotyping of non-template controls (to ensure the absence of cross-contamination and primer crossreactivity) and genotyping of samples set as technical replicates to evaluate consistency. There was $100 \%$ successful genotyping call rate. Re-genotyping of $25 \%$ of the samples demonstrated $100 \%$ concordance.

Analysis Statistical analyses were performed using the $\mathrm{R}$ v3.3.2 statistical software (within RStudio v0.99.902; www. rstudio.com). Univariable analysis was undertaken to compare clinical and biochemical risk factors among women with and without GDM and by CREBRF status (G/G vs G/A or $\mathrm{A} / \mathrm{A})$, using $\chi^{2}$ test for categorical data and $t$ test for continuous data. Logistic regression was used to test for an association between rs373863828 minor allele (c.1370A, p. 457Gln) and GDM. Māori and Pacific women were 
analysed separately and the effects were combined using an inverse-variance-weighted fixed-effect meta-analysis. Heterogeneity among sample sets was assessed using Cochran's heterogeneity $(Q)$ statistics. A $p$ value $<0.05$ in meta-analyses was considered statistically significant.

Results are presented as allelic ORs with $95 \%$ CIs, representing the estimated effect of each copy of the minor A allele on likelihood of GDM. Multivariable analysis was carried out to adjust for potential confounding (maternal age, BMI and family history of diabetes) and mediating factors (weekly weight change from recruitment to 36 weeks and $\mathrm{HbA}_{1 \mathrm{c}}, \mathrm{HDL}-\mathrm{cholesterol}$ and triacylglycerol concentrations at recruitment). None of the models adjusted for the HUMBA trial treatments. The predictive value of early pregnancy risk factors (maternal age, BMI and family history of diabetes) for GDM with and without $C R E B R F$ rs 373863828 genotype status (A/G or $\mathrm{A} / \mathrm{A})$ was evaluated by comparing the area under the corresponding receiver operating characteristic (ROC) curves. Specificity, sensitivity, positive and negative predictive values and the negative likelihood ratio were calculated using the DAG stats software package (v2000, https:// biostats.com.au/DAG_Stat) [20].

\section{Results}

Of 230 women with obesity in the HUMBA trial, 166 (72\%) were of Māori or Pacific ethnicity; 112 (67\%) completed both OGTT and $C R E B R F$ testing and were included in the analysis (Fig. 1). The $C R E B R F$ rs 373863828 (A/G or A/A) allele was carried by $31(28 \%)$ women: 28/77 (36\%) of the women

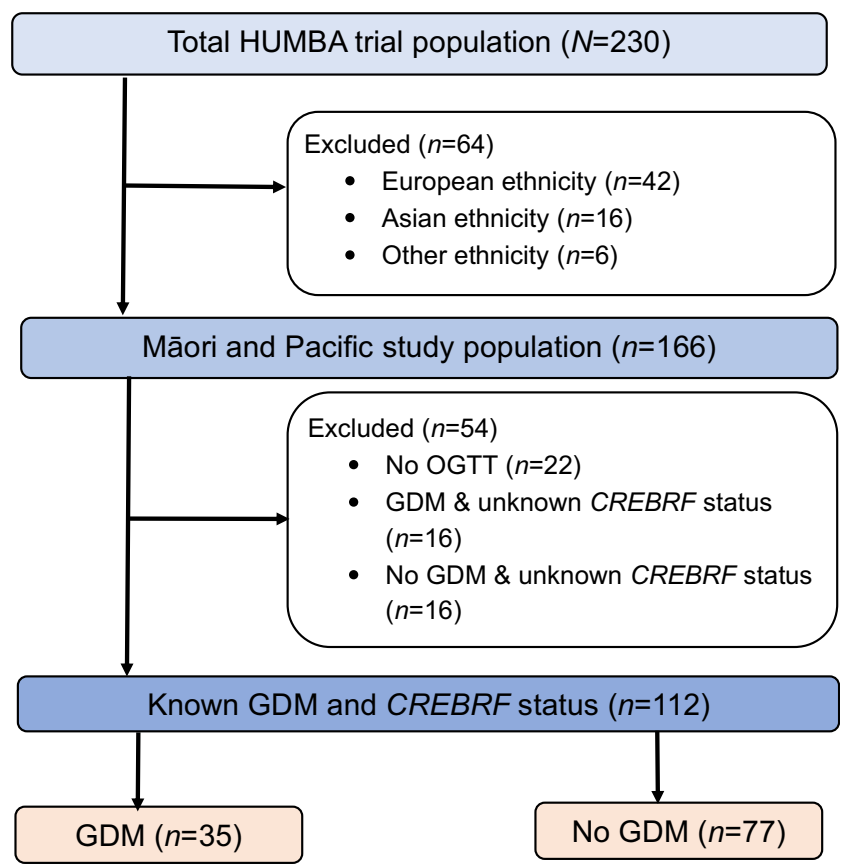

Fig. 1 Participant flow in the $C R E B R F$ study without GDM and 3/35 (9\%) of the women with GDM. GDM was diagnosed in 35 (31\%) women: $3 / 27(11 \%)$ women with the A/G genotype and 32/81 (40\%) women with the G/G genotype (Table 1); none of the four women with the $\mathrm{A} / \mathrm{A}$ genotype developed GDM. In univariable analyses, women who developed GDM had higher BMI $(p=0.02)$, waist circumference $(p=0.03)$, and $\mathrm{HbA}_{1 \mathrm{c}}$ level at recruitment $(p<0.001)$ and higher fasting glucose concentration $(p<0.001)$ and $1 \mathrm{~h}$ and $2 \mathrm{~h}$ glucose concentrations (both $p<0.001$ ) on OGTT (Table 1 ). There were no statistically significant differences in clinical and biochemical risk factors between women with and without the CREBRF rs 373863828 (A) allele; carriers had lower fasting and $2 \mathrm{~h}$ glucose concentrations on OGTT (Table 1) but the differences did not reach statistical significance ( $p=0.05$ and $p=0.07$, respectively).

The CREBRF rs373863828 (A) allele was associated with a statistically significant decrease in the likelihood of developing GDM in both univariable analysis (OR 0.19 [95\% CI $0.05,0.69], p=0.01$ ) and after adjustment for potential confounding by maternal age, BMI and family history of diabetes (adjusted OR 0.13 [95\% CI 0.03, 0.53], $p=0.004$ ) (Table 2). Adjustment for potential mediators, including GWG and early gestational $\mathrm{HbA}_{1 \mathrm{c}}$, HDL-cholesterol and triacylglycerol concentrations, did not alter the association between CREBRF rs373863828 (A) allele and GDM (adjusted OR 0.18 [95\% CI 0.04, 0.74], $p=0.02$ ).

In ROC analysis, the AUC for clinical risk factors (maternal age, BMI and family history of diabetes) was 0.67 (95\% CI $0.57,0.78$ ) (Fig. 2). Addition of rs 373863828 status (A/G or $\mathrm{A} / \mathrm{A}$ ) increased the predictive value of the model, giving an AUC of 0.76 (95\% CI $0.67,0.85$ ), an absolute increase of $8.8 \%$ (95\% CI 1.0, 16.6, $p=0.03$ ) (Fig. 2). Table 3 provides estimates of the specificity, sensitivity and positive and negative predictive value and negative likelihood ratio of rs373863828 status for GDM. Overall, the sensitivity and specificity of rs 373863828 status for GDM was $91 \%$ and $36 \%$, respectively, with a positive predictive value $(\mathrm{G} / \mathrm{G})$ of $40 \%$, a negative predictive value $(\mathrm{A} / \mathrm{G}$ or $\mathrm{A} / \mathrm{A})$ of $90 \%$ and a negative likelihood ratio of 0.24 .

\section{Discussion}

We have identified a novel protective biomarker, the minor (A) allele of $C R E B R F$ rs 373863828 , that is associated with a fivefold reduction in the likelihood of GDM (defined by IADPSG criteria) in Māori and Pacific women with obesity. This is an important finding, given the high rates of GDM and obesity in these ethnic groups, and the fact that GDM is a major risk factor for subsequent development of type 2 diabetes in women [12].

This decreased rate of GDM in women carrying the $C R E B R F$ rs373863828 minor (A) allele appeared to be due 
Table 1 Cohort characteristics

\begin{tabular}{|c|c|c|c|c|c|c|c|}
\hline \multirow[t]{2}{*}{ Characteristic } & \multirow[t]{2}{*}{ Total $(N=112)$} & \multicolumn{3}{|l|}{ GDM } & \multicolumn{3}{|c|}{$C R E B R F$ rs 373863828 (A) allele } \\
\hline & & No $(N=77)$ & Yes $(N=35)$ & $p$ value & No $(N=81)$ & Yes $(N=31)$ & $p$ value \\
\hline \multicolumn{8}{|l|}{ Ethnicity, $n(\%)$} \\
\hline Māori & $37(33.0)$ & $31(40.3)$ & $6(17.1)$ & \multirow[t]{2}{*}{0.02} & $24(29.6)$ & $13(41.9)$ & \multirow[t]{2}{*}{0.22} \\
\hline Pacific & $75(67.0)$ & $46(59.7)$ & $29(82.9)$ & & $57(70.4)$ & $18(58.1)$ & \\
\hline Maternal age, years & $29.0(6.1)$ & $28.8(6.1)$ & $29.4(6.2)$ & 0.67 & $29.3(6.3)$ & $28.3(5.7)$ & 0.43 \\
\hline \multicolumn{8}{|l|}{ Family medical history, $n(\%)$} \\
\hline Hypertension $^{\mathrm{a}}$ & $55(52.4)$ & $38(52.8)$ & $17(51.5)$ & 0.91 & $37(48.1)$ & $18(64.2)$ & 0.14 \\
\hline Diabetes $^{\mathrm{b}}$ & $44(40.4)$ & $28(36.8)$ & $16(48.5)$ & 0.27 & $34(43.0)$ & $10(33.3)$ & 0.35 \\
\hline \multicolumn{8}{|l|}{ Body size at recruitment } \\
\hline Height, cm & $167.6(5.2)$ & $168.0(5.5)$ & $166.8(4.4)$ & 0.22 & $167.4(5.1)$ & $168.2(5.5)$ & 0.44 \\
\hline Weight, kg & $110.8(18.4)$ & $108.7(18.3)$ & $115.5(18.1)$ & 0.07 & $109.6(17.4)$ & $113.9(20.8)$ & 0.31 \\
\hline BMI, $\mathrm{kg} / \mathrm{m}^{2}$ & $39.4(6.4)$ & $38.5(6.1)$ & $41.5(6.6)$ & 0.02 & $39.1(6.0)$ & $40.2(7.3)$ & 0.45 \\
\hline Waist circumference, $\mathrm{cm}$ & $115.8(12.8)$ & $114.1(12.8)$ & $119.6(11.9)$ & 0.03 & $115.0(11.9)$ & $117.9(14.9)$ & 0.33 \\
\hline \multicolumn{8}{|l|}{ Metabolic status at recruitment } \\
\hline $\mathrm{HbA}_{1 \mathrm{c}}, \mathrm{mmol} / \mathrm{mol}$ & $35.3(4.0)$ & $34.2(3.4)$ & $37.7(4.0)$ & \multirow[t]{2}{*}{$<0.001$} & $35.4(4.1)$ & $35.0(3.5)$ & \multirow[t]{2}{*}{0.56} \\
\hline $\mathrm{HbA}_{1 \mathrm{c}}, \%$ & $5.4(0.6)$ & $5.3(0.5)$ & $5.5(0.6)$ & & $5.4(0.6)$ & $5.4(0.5)$ & \\
\hline Total cholesterol, mmol/1 & $4.5(0.7)$ & $4.5(0.7)$ & $4.6(0.8)$ & 0.78 & $4.5(0.7)$ & $4.5(0.7)$ & 0.77 \\
\hline Triacylglycerol, mmol// $\mathrm{l}^{\mathrm{c}}$ & $2.1(0.6)$ & $2.1(0.6)$ & $2.1(0.6)$ & 0.61 & $2.1(0.6)$ & $2.2(0.6)$ & 0.47 \\
\hline HDL-cholesterol, mmol// $\mathrm{l}^{\mathrm{a}}$ & $1.5(0.3)$ & $1.5(0.3)$ & $1.5(0.3)$ & 0.37 & $1.5(0.3)$ & $1.5(0.3)$ & 0.88 \\
\hline LDL-cholesterol, mmol/1 ${ }^{\mathrm{a}}$ & $2.1(0.8)$ & $2.1(0.7)$ & $2.1(0.9)$ & 0.93 & $2.1(0.8)$ & $2.0(0.7)$ & 0.34 \\
\hline \multicolumn{8}{|l|}{ HUMBA trial treatments, $n(\%)$} \\
\hline Dietary intervention & $57(50.9)$ & $39(50.7)$ & $18(51.4)$ & \multirow[t]{2}{*}{0.93} & $42(51.9)$ & $15(48.4)$ & \multirow[t]{2}{*}{0.74} \\
\hline Routine diet advice & $55(49.1)$ & $38(49.4)$ & $17(48.6)$ & & $39(48.1)$ & $16(51.6)$ & \\
\hline Probiotics & $61(54.5)$ & $43(55.8)$ & $18(51.4)$ & \multirow[t]{2}{*}{0.66} & $42(51.9)$ & $19(61.3)$ & \multirow[t]{2}{*}{0.37} \\
\hline Placebo & $51(45.5)$ & $34(44.2)$ & $17(48.6)$ & & $39(48.1)$ & $12(38.7)$ & \\
\hline \multicolumn{8}{|l|}{ GWG, kg } \\
\hline Mean weekly change recruitment to 28 weeks $^{\mathrm{d}}$ & $0.51(0.32)$ & $0.52(0.34)$ & $0.49(0.28)$ & 0.70 & $0.51(0.34)$ & $0.52(0.30)$ & 0.90 \\
\hline Mean weekly change recruitment to 36 weeks $^{\mathrm{a}}$ & $0.56(0.31)$ & $0.58(0.34)$ & $0.53(0.25)$ & 0.38 & $0.54(0.32)$ & $0.61(0.30)$ & 0.27 \\
\hline Hypertension in pregnancy, $n(\%)$ & $15(13.4)$ & $10(13.0)$ & $5(14.3)$ & 0.86 & $11(13.6)$ & $4(12.9)$ & 0.93 \\
\hline \multicolumn{8}{|l|}{ OGTT glucose concentration, mmol/1 } \\
\hline Fasting & $4.7(0.5)$ & $4.4(0.3)$ & $5.2(0.5)$ & $<0.001$ & $4.7(0.6)$ & $4.5(0.4)$ & 0.05 \\
\hline $1 \mathrm{~h}$ & $8.0(1.8)$ & $7.2(1.3)$ & $9.6(1.6)$ & $<0.001$ & $8.1(1.9)$ & $7.8(1.7)$ & 0.49 \\
\hline $2 \mathrm{~h}$ & $6.2(1.2)$ & $5.8(0.9)$ & $7.0(1.4)$ & $<0.001$ & $6.3(1.2)$ & $5.8(1.2)$ & 0.07 \\
\hline \multicolumn{8}{|l|}{$C R E B R F$ genotype, $n(\%)$} \\
\hline $\mathrm{G} / \mathrm{G}$ & $81(72.3)$ & $49(63.6)$ & $32(91.4)$ & 0.002 & & & \\
\hline $\mathrm{A} / \mathrm{G}$ & $27(24.1)$ & $24(31.2)$ & $3(8.6)$ & 0.01 & & & \\
\hline $\mathrm{A} / \mathrm{A}$ & $4(3.6)$ & $4(5.2)$ & $0(0.0)$ & & & & \\
\hline Minor (A) allele & 35 (15.6) & $32(20.8)$ & $3(4.3)$ & 0.002 & & & \\
\hline
\end{tabular}

Data are $n(\%)$ or mean $(\mathrm{SD})$

$p$ values are for the comparison $\left(\chi^{2}\right.$ or $t$ test) between women who have vs do not have GDM or who do vs do not carry the CREBRF rs373863828 minor (A) allele

${ }^{a}$ Data missing for 7 participants

${ }^{\mathrm{b}}$ Data missing for 3 participants

${ }^{\mathrm{c}}$ Data missing for 6 participants

${ }^{\mathrm{d}}$ Data missing for 9 participants

to a reduction in both fasting and $2 \mathrm{~h}$ OGTT glucose concentrations. Adjustment for potential confounding strengthened this association, suggesting that the CREBRF rs 373863828 minor (A) allele is associated with reduced likelihood of 
Table 2 Association between CREBRF rs373863828 (A) allele and risk of GDM in Māori and Pacific women

\begin{tabular}{|c|c|c|c|c|c|c|c|c|c|}
\hline \multirow[t]{2}{*}{ Population } & \multicolumn{3}{|c|}{ Model $1^{\mathrm{a}}$} & \multicolumn{3}{|c|}{ Model $2^{\mathrm{b}}$} & \multicolumn{3}{|c|}{ Model $3^{c}$} \\
\hline & $\mathrm{N}$ & OR $(95 \% \mathrm{CI})$ & $p$ value & $\mathrm{N}$ & OR $(95 \% \mathrm{CI})$ & $p$ value & $\mathrm{N}$ & OR $(95 \% \mathrm{CI})$ & $p$ value \\
\hline Total & 112 & $0.19(0.049,0.69)$ & 0.012 & 112 & $0.13(0.033,0.53)$ & 0.004 & 102 & $0.18(0.042,0.74)$ & 0.018 \\
\hline Māori & 37 & $0.33(0.040,2.73)$ & 0.30 & 37 & $0.19(0.012,2.85)$ & 0.23 & 33 & $0.17(0.01,3.01)$ & 0.23 \\
\hline Pacific & 75 & $0.15(0.033,0.69)$ & 0.015 & 75 & $0.12(0.023,0.59)$ & 0.009 & 69 & $0.18(0.034,0.93)$ & 0.04 \\
\hline
\end{tabular}

${ }^{\text {a }}$ Model 1 is unadjusted

${ }^{\mathrm{b}}$ Model 2 adjusts for potential confounding by maternal age, BMI at recruitment and family history of diabetes

${ }^{\mathrm{c}}$ Model 3 adjusts for potential mediation by mean weekly weight change from baseline to 36 weeks' gestation, and HbA $\mathrm{Ac}_{1 \mathrm{c}}$, HDL-cholesterol and triacylglycerol concentrations at recruitment

GDM independent of maternal age, BMI and family history of diabetes. Adjustment for GWG and $\mathrm{HbA}_{1 \mathrm{c}}$, HDL-cholesterol and triacylglycerol concentrations in early gestation did not alter the association between the $C R E B R F$ rs373863828 (A) minor allele and GDM, suggesting that these are not mediating factors.

The $C R E B R F$ gene is found on chromosome 5 and encodes a negative regulatory factor of the cyclic AMP-responsive element-binding protein 3 (CREB3), which in turn is involved in regulation of protein translation. The rs373863828 (A) allele variant was first associated with higher BMI $\left(1.5 \mathrm{~kg} / \mathrm{m}^{2}\right)$ and waist circumference $(3 \mathrm{~cm})$ but lower odds of type 2 diabetes (OR 0.59) among people of Polynesian ancestry [13, 14, 21]. The minor (A) allele was also weakly associated with increased insulin sensitivity by HOMA-IR in the Sāmoan and American Sāmoan populations [13]. The protective effect of the minor (A) allele for GDM is concordant with the findings of previous association studies among non-pregnant women, which

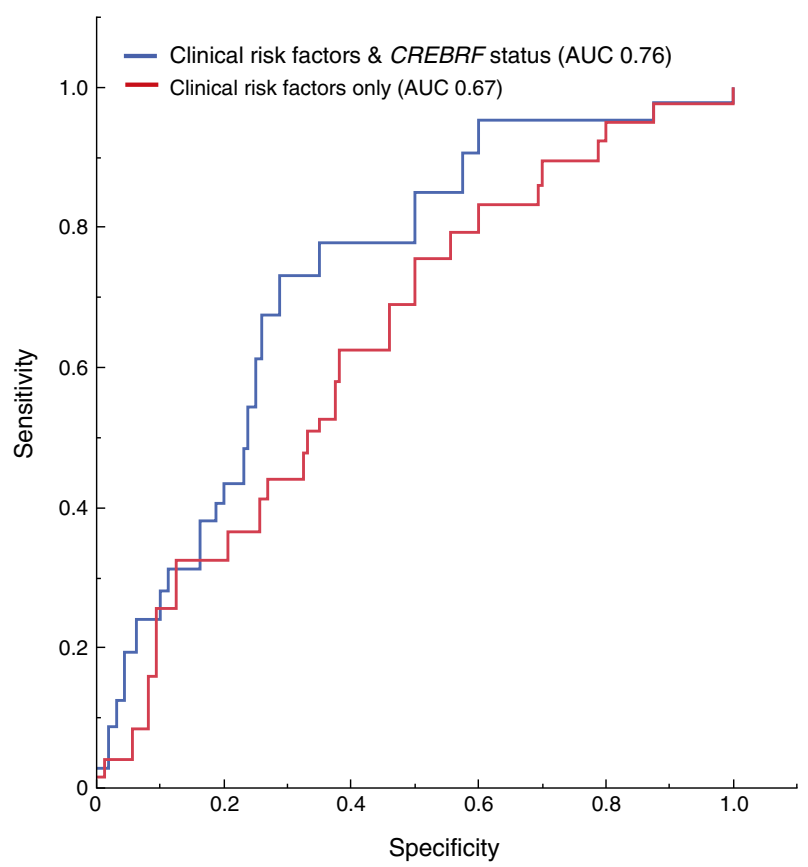

Fig. 2 ROC curves for prediction of GDM. Clinical risk factors include maternal age, BMI and family history of diabetes suggested a strong genetic relationship between the $C R E B R F$ A allele and lower risk of both GDM and type 2 diabetes.

In this cohort of pregnant women with obesity, when comparing those with and without the minor (A) allele there were no statistically significant differences in BMI, waist circumference or GWG. We speculate that the influence of the CREBRF rs 373863828 variant may be associated with increased lean mass rather than any changes in adiposity manifested as GWG. CREBRF rs373863828 variant carriers have been shown to be taller $[22,23]$ and hence height-related increase in muscle mass and pancreatic beta cell mass may decrease the risk of GDM and type 2 diabetes without influencing GWG.

It remains unclear how the rs373863828 minor (A) allele contributes to higher BMI yet lower risk of type 2 diabetes. Cellular models have suggested that the minor (A) allele promotes lipid storage at a reduced energy cost in the adipocytes [13], although this does not explain the lower risk of type 2 diabetes or GDM. CREBRF may also have a role in modifying fat distribution (subcutaneous vs abdominal fat), which may influence flux of NEFA and disposal of glucose [24]. Interestingly, $C R E B R F$-knockout mice have shown markedly reduced prolactin secretion and augmented glucocorticoid receptor signalling [25]. Given the key role of prolactin in the adaptation of insulin-secreting beta cells during pregnancy [26], and the known diabetogenic effects of glucocorticoids [27], this suggests that $C R E B R F$ has a role in adaptive changes in the pancreas and in the regulation of insulin resistance during pregnancy. However, further investigation of beta cell function, insulin sensitivity and glucose disposal levels are needed to fully understand the effect of the CREBRF rs373863828 minor (A) allele on glucose metabolism during pregnancy. Elucidating the underlying molecular mechanisms may provide opportunity for development of novel therapeutic interventions for GDM, particularly as $C R E B R F$ has been considered as a target gene in other conditions, such as gastric cancer and glioblastoma [28, 29].

In this population, detection of the CREBRF rs 373863828 minor (A) allele had high $(90 \%)$ negative predictive value for GDM, giving a negative likelihood ratio of 0.24 . Although not 
Table 3 Predictive value of the $C R E B R F$ status for GDM

\begin{tabular}{lrlllll}
\hline Population & $N$ & Sensitivity & Specificity & PPV & NPV & Negative likelihood ratio $^{\text {a }}$ \\
\hline Total & 112 & $0.91(0.77,0.98)$ & $0.36(0.26,0.48)$ & $0.40(0.29,0.51)$ & $0.90(0.74,0.98)$ & $0.24(0.08,0.72)$ \\
Māori & 37 & $0.83(0.36,1.00)$ & $0.39(0.22,0.58)$ & $0.21(0.07,0.42)$ & $0.92(0.64,1.00)$ & $0.43(0.07,2.72)$ \\
Pacific & 75 & $0.93(0.77,0.99)$ & $0.35(0.21,0.50)$ & $0.47(0.34,0.61)$ & $0.89(0.65,0.99)$ & $0.20(0.05,0.80)$ \\
\hline
\end{tabular}

Data are probability $(95 \% \mathrm{CI})$ or likelihood ratio $(95 \% \mathrm{CI})$

$C R E B R F$ wild type $(\mathrm{G} / \mathrm{G})$ considered as positive screen and $C R E B R F$ rs373863828 variant $(\mathrm{A} / \mathrm{G}$ or $\mathrm{A} / \mathrm{A})$ considered as negative screen

${ }^{a}$ Likelihood ratios of 0.1 have a large effect on post-test probability of disease, ratios of 0.2 have a moderate effect and ratios of 0.5 a slight effect [34] NPV, negative predictive value; PPV, positive predictive value

sufficiently predictive to replace diagnostic testing for GDM [30], the CREBRF rs373863828 genotype could be used in Māori and Pacific women for risk stratification in early pregnancy, and there is increasing evidence that risk prediction models need to incorporate ethnic-specific biomarkers [31]. Māori and Pacific women carrying the minor allele exhibited a low incidence of GDM, despite clinically increased risk due to obesity. Given that $60 \%$ of Māori and Pacific women in the Counties Manukau region in New Zealand have obesity and nearly $30 \%$ carry the protective minor (A) allele, genotyping may have clinical utility by allowing targeting of resources for women at higher risk of GDM (absence of minor [A] allele). This could include preventative dietary interventions and pharmacotherapy, and earlier testing for GDM by OGTT, rather than by the current two-step GDM screening used in New Zealand (OGTT is reserved for women with an abnormal non-fasting polycose test). These data have relevance not only for Aotearoa/New Zealand but also for the Pacific Islands, which have even higher rates of type 2 diabetes and GDM [32].

Although the magnitude of association that we observed between the CREBRF rs373863828 minor (A) allele and GDM was large and not obviously confounded, the fact that our study was conducted only in women with obesity raises the possibility of selection bias. However, typical collider conditioning is unlikely as this requires both the exposure (CREBRF rs373863828 genotype) and outcome (GDM) to be causally linked to sample selection (obesity), and this does not seem plausible in the case of GDM. Nevertheless, replication of our findings in a broader population is warranted, particularly as we used meta-analysis of self-reported ethnicity to estimate exposure effects rather than genetic population structure estimates.

Overall, the combination of $C R E B R F$ rs373863828 genotype and clinical risk factors had greater predictive value for GDM than clinical risk factors alone. We were unable to explore whether there is an interaction between $C R E B R F$ rs373863828 genotype and clinical risk factors for prediction of GDM due to the limited sample size of our study. It is possible that clinical risk prediction may perform better in
Māori and Pacific women with the rs373863828 GG genotype, allowing further optimisation of risk stratification, but this would require evaluation in larger cohorts, including women without obesity. Identifying ways to prevent development of GDM among Māori and Pacific women is a health priority [33].

In summary, we have shown that the CREBRF rs373863828 minor (A) allele is associated with substantially reduced likelihood of GDM in Māori and Pacific women with obesity. The CREBRF rs373863828 genotype could potentially improve risk stratification for GDM in early pregnancy in Māori and Pacific women.

Acknowledgements We wish to thank the women who participated in the HUMBA trial. We also acknowledge the HUMBA trial research midwives C. O'Driscoll, S. Va'afusuaga, S. Ross-Heard and A. Hallaran, and research nurse M. McCowan for the collection of data and specimens, from the University of Auckland. Some of the data were presented as an abstract at the 23rd Annual Congress of the Perinatal Society of Australia and New Zealand (PSANZ) meeting in 2019.

Data availability Published data are available to approved researchers under the data sharing arrangements provided by the Clinical Data Research Hub (CDRH), based at the Liggins Institute, University of Auckland (https://wiki.auckland.ac.nz/researchhub). Metadata, along with instructions for data access, are available at the University of Auckland's research data repository, Figshare (https://auckland.figshare. com). Data access requests are to be submitted to the Data Access Committee via researchhub@auckland.ac.nz. De-identified published data will be shared with researchers who provide a methodologically sound proposal and have appropriate ethical and institutional approval. Researchers must sign and adhere to the Data Access Agreement, which includes a commitment to using the data only for the specified proposal, to refrain from any attempt to identify individual participants, to store data securely and to destroy or return the data after completion of the project. The CDRH reserves the right to charge a fee to cover the costs of making data available, if required.

Funding Funding for this study was provided by the Health Research Council of New Zealand and Cure Kids, New Zealand. The funders had no role in study design, data collection, analysis or the decision to publish.

Authors' relationships and activities The authors declare that there are no relationships or activities that might bias, or be perceived to bias, their work. 
Contribution statement MK, RM, JT, LM, KOG, RT and CM planned the study. KOG, RT, LM and CM supervised data collection. MK, TM and MJ performed genotyping. MK, JT and CM conducted analyses. MK and $\mathrm{CM}$ drafted the manuscript. All authors contributed to the discussion, critically appraised the manuscript and approved the final version for publication. CM and RM are the guarantors for this work and accept full responsibility for the conduct of the study, had access to the data, and controlled the decision to publish.

\section{References}

1. Kampmann U, Madsen LR, Skajaa GO, Iversen DS, Moeller N, Ovesen P (2015) Gestational diabetes: a clinical update. World J Diabetes 6(8):1065-1072

2. Lain KY, Catalano PM (2007) Metabolic changes in pregnancy. Clin Obstet Gynecol 50(4):938-948

3. Barbour LA, McCurdy CE, Hernandez TL, Kirwan JP, Catalano PM, Friedman JE (2007) Cellular mechanisms for insulin resistance in normal pregnancy and gestational diabetes. Diabetes Care 30(Suppl 2):S112-S119

4. Metzger BE, Gabbe SG, Persson B et al (2010) International Association of Diabetes and Pregnancy Study Groups recommendations on the diagnosis and classification of hyperglycemia in pregnancy. Diabetes Care 33(3):676-682

5. Dias S, Pheiffer C, Abrahams Y, Rheeder P, Adam S (2018) Molecular biomarkers for gestational diabetes mellitus. Int J Mol Sci 19(10):2926

6. Sladek R, Rocheleau G, Rung J et al (2007) A genome-wide association study identifies novel risk loci for type 2 diabetes. Nature 445(7130):881-885

7. Zeggini E, Scott LJ, Saxena R et al (2008) Meta-analysis of genomewide association data and large-scale replication identifies additional susceptibility loci for type 2 diabetes. Nat Genet 40(5):638-645

8. Scott RA, Scott LJ, Magi R et al (2017) An expanded genome-wide association study of type 2 diabetes in Europeans. Diabetes 66(11): 2888-2902

9. Kwak SH, Kim SH, Cho YM et al (2012) A genome-wide association study of gestational diabetes mellitus in Korean women. Diabetes 61(2):531-541

10. Huopio H, Cederberg H, Vangipurapu J et al (2013) Association of risk variants for type 2 diabetes and hyperglycemia with gestational diabetes. Eur J Endocrinol 169(3):291-297

11. Cho YM, Kim TH, Lim S et al (2009) Type 2 diabetes-associated genetic variants discovered in the recent genome-wide association studies are related to gestational diabetes mellitus in the Korean population. Diabetologia 52(2):253-261

12. Bellamy L, Casas JP, Hingorani AD, Williams D (2009) Type 2 diabetes mellitus after gestational diabetes: a systematic review and meta-analysis. Lancet 373(9677):1773-1779

13. Minster RL, Hawley NL, Su CT et al (2016) A thrifty variant in $C R E B R F$ strongly influences body mass index in Samoans. Nat Genet 48(9):1049-1054

14. Krishnan M, Major TJ, Topless RK et al (2018) Discordant association of the $C R E B R F$ rs373863828 A allele with increased BMI and protection from type 2 diabetes in Maori and Pacific (Polynesian) people living in Aotearoa/New Zealand. Diabetologia 61(7):1603-1613

15. Hanson RL, Safabakhsh S, Curtis JM et al (2019) Association of $C R E B R F$ variants with obesity and diabetes in Pacific Islanders from Guam and Saipan. Diabetologia 62(9):1647-1652

16. Okesene-Gafa KAM, Li M, McKinlay CJD et al (2019) Effect of antenatal dietary interventions in maternal obesity on pregnancy weight-gain and birthweight: Healthy Mums and Babies (HUMBA) randomized trial. Am J Obstet Gynecol 221(2): 152.e1-152.e13

17. Ministry of Health (2014) Screening, diagnosis and management of gestational diabetes in New Zealand. A clinical practice guideline. Ministry of Health, Wellington

18. Rasmussen K, Yaktine A (2009) Weight gain during pregnancy: reexamining the guidelines. The National Academies Press, Washington

19. Culliney K, McCowan LME, Okesene-Gafa K et al (2018) Accuracy of point-of-care HbAlc testing in pregnant women. Aust N Z J Obstet Gynaecol 58(6):643-647. https://doi.org/10. 1111/ajo.12786

20. Mackinnon A (2000) A spreadsheet for the calculation of comprehensive statistics for the assessment of diagnostic tests and interrater agreement. Comput Biol Med 30(3):127-134

21. Naka I, Furusawa T, Kimura R et al (2017) A missense variant, rs373863828-A (p.Arg457Gln), of CREBRF and body mass index in Oceanic populations. J Hum Genet 62(9):847-849

22. Carlson JC, Rosenthal SL, Russell EM et al (2020) A missense variant in $C R E B R F$ is associated with taller stature in Samoans. Am J Hum Biol. https://doi.org/10.1002/ajhb.23414

23. Metcalfe LK, Krishnan M, Turner N et al (2019) The Maori and Pacific specific CREBRF variant and adult height. Int J Obes 44: 748-752. https://doi.org/10.1038/s41366-019-0437-6

24. Loos RJF, Kilpelainen TO (2018) Genes that make you fat, but keep you healthy. J Intern Med 284(5):450-463

25. Martyn AC, Choleris E, Gillis DJ et al (2012) Luman/CREB3 recruitment factor regulates glucocorticoid receptor activity and is essential for prolactin-mediated maternal instinct. Mol Cell Biol 32(24):5140-5150

26. Banerjee RR, Cyphert HA, Walker EM et al (2016) Gestational diabetes mellitus from inactivation of prolactin receptor and MafB in islet $\beta$-cells. Diabetes 65(8):2331-2341

27. Di Dalmazi G, Pagotto U, Pasquali R, Vicennati V (2012) Glucocorticoids and type 2 diabetes: from physiology to pathology. J Nutr Metab 2012:525093

28. Xue H, Zhang J, Guo X et al (2016) CREBRF is a potent tumor suppressor of glioblastoma by blocking hypoxia-induced autophagy via the CREB3/ATG5 pathway. Int J Oncol 49(2):519-528

29. Han J, Zhang L, Zhang J et al (2018) CREBRF promotes the proliferation of human gastric cancer cells via the AKT signaling pathway. Cell Mol Biol 64(5):40-45

30. Deeks JJ, Altman DG (2004) Diagnostic tests 4: Likelihood ratios. BMJ 329(7458):168-169

31. Sweeting AN, Wong J, Appelblom H et al (2019) A novel early pregnancy risk prediction model for gestational diabetes mellitus. Fetal Diagn Ther 45(2):76-84

32. Moy KL, Sallis JF, David KJ (2010) Health indicators of Native Hawaiian and Pacific Islanders in the United States. J Community Health 35(1):81-92

33. Reid J, Anderson A, Cormack D, Reid P, Harwood M (2018) The experience of gestational diabetes for indigenous Maori women living in rural New Zealand: qualitative research informing the development of decolonising interventions. BMC Pregnancy Childbirth 18(1):478

34. McGee S (2002) Simplifying likelihood ratios. J Gen Intern Med 17(8):646-649

Publisher's note Springer Nature remains neutral with regard to jurisdictional claims in published maps and institutional affiliations. 